\title{
Study and Improvement of Iris Location Algorithm
}

\author{
Caitang Sun, Chunguang Zhou*, Yanchun Liang, and Xiangdong Liu \\ College of Computer Science and Technology, \\ Jilin University, Changchun, 130012, China \\ cgzhouljlu.edu.cn
}

\begin{abstract}
Iris location is a crucial step in iris recognition. Taking into consideration the fact that interior of the pupil, there would have some lighter spots because of reflection, this paper improves the commonly used coarse location method. It utilizes the gray scale histogram of the iris graphics, first computes the binary threshold, averaging the center of chords to coarsely estimate the center and radius of the pupil, and then finely locates it using the algorithm of circle detection in the binary graphic. This method could reduce the error of locating within the pupil. After that, this paper combines Canny edge detector and Hough voting mechanism to locate the outer boundary. Finally, a statistical method is exploited to exclude eyelash and eyelid areas. Experiments have shown the applicability and efficiency of this algorithm.
\end{abstract}

Keywords: Iris Location, Circle Detection, Canny Edge Detection, Hough Voting Mechanism.

\section{Introduction}

Iris recognition has become an important solution for individual identification. As an emerging biometric recognition technology, it has some advantages compared to others: (1) It is impossible that any two individual's texture of iris is complete the same, and even the left and the right one of the same individual are also different from each other; (2) The features of the iris are changeless during one's lifetime without any accident; (3) Unlike other information such as face and password, it is difficult to change or simulate. All these advantages make it a hot topic.

Iris location aims at locating the inner boundary (pupil) and outer one (sclera) of the iris, providing valid areas for iris feature extraction, which could directly influence the effect of iris recognition. There are two most commonly used iris location algorithms. One is the circle-detection algorithm proposed by J.Daugman[14], which uses circular edge detecting operator to detect the inner and outer boundary of the iris, exploiting the geometrical characteristic that the iris is approximately a circle. And the other one is the two-step method proposed by P.Wildes[5]. Cui Jiali etc[6] combine SVM and LDA for iris location, but it may be influenced if the eyelashes are heavy; Yuan Weiqi etc[7] present an active contour method-SnakeDaugman, and it could also be influenced by eyelashes. Most of the iris location algorithms coarsely locate pupil by finding the minimum of the sum of gray value

* Corresponding author. 
before fine location, because the gray level of pupil is lower than that of all the other areas in the iris image. But the disadvantage is also obvious, i.e., if the gray level of some pixels in the horizontal or vertical direction of the true pupil center is made higher or others made lower because of some factors such as lightness, the result will be far away from the actual position. Xue Bai etc [8] use histogram to compute the threshold for binarization, and it improves the effect of iris location in certain extent, but in some conditions, the gray level of eyelash or eyebrow could be lower than that of the pupil, so the threshold would be so low that the result is not ideal. This paper improves the method in coarse location of the pupil, uses binary image circle detector, and combines edge detection and Hough voting mechanism in outer boundary detection $[5,9,10]$. Experiments show that the effect is satisfactory.

The remainder of this paper is organized as follows. Section 2.1 mainly introduces the coarse location method. Section 2.2 depicts the fine location method of the inner boundary. Section 2.3 describes the fine location method for the outer boundary. Section 3 introduces how to exclude eyelid and eyelash area from the result above. Section 4 presents experimental results and concludes with some remarks.

\section{Iris Location}

\subsection{Inner Boundary (Pupil) Coarse Location}

The objective of pupil coarse location is to approximately estimate the center and radius of the pupil, that is, to determine the pseudo center and pseudo radius of it. In general, in an iris image, the gray values inside the pupil are the lowest in the image. Whereas, the gray values of eyelashes and eyebrows are often near to those of the pupil, or even lower than them in some conditions.

In this paper, the image is binarized first. Selection of the threshold is crucial, which influences the following steps. If the threshold is too low, the area of pupil would be reduced, vice versa. Based on this analysis, this paper proposed the following method.

(1) Make the gray scale histogram of the image, then filter it, and compute the valley between the first two wave peaks, whose gray value is marked as $T_{0}$. In some instances, it could obtain good result if $\mathrm{T}_{0}$ is directly used as the threshold. But, in many images, the gray level of eyelash or eyebrow is lower than that of the pupil, in these cases, the calculated threshold will be lower than the needed one, and pupil would be mistaken for background, so, further judgement is necessary;

(2) Calculate the difference between $T_{0}$ and the first wave peak, if it is larger than $6, \mathrm{~T}_{0}$ could be taken as $\mathrm{T}_{1}$, the ultimate threshold; otherwise, continue to search for the valley after the third wave peak, and select the correspond gray value as $\mathrm{T}_{1}$ (Fig.1(b));

(3) Binarize the image, set the values of the pixels whose gray level below $\mathrm{T}_{1}$ to 0 , others to 255 ;

In some cases, there will still have some noises in the binary image because of the existence of eyelash or eyebrow (Fig.1(c)), but most of them could be removed by the Open and Close operation of morphology. 

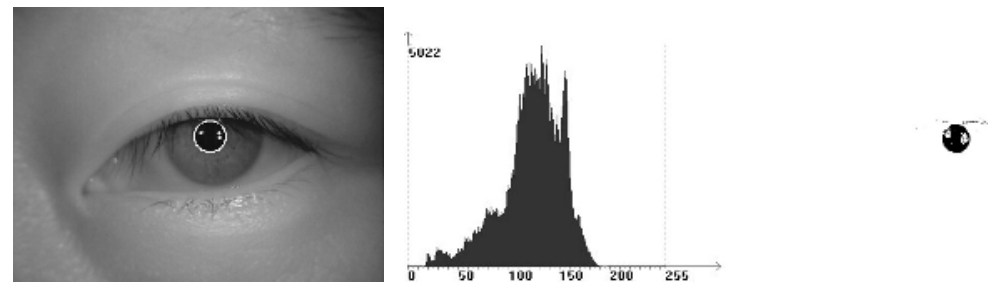

Fig. 1. (a) Location result of the inner boundary (b) histogram of the image with $T_{1}=25$ binary image

Then summarize the gray value in the direction of $\mathrm{x}$ and $\mathrm{y}$ respectively, and find the point correspond to the minimum of them. This point may be near to the center of pupil, but it is also possible to be far away from it, so, it is necessary for further determination (In fact, because the most time consuming in iris location is fine location of the inner and outer boundary, while the result of coarse location will determine the time and effect of fine location, it is worthy of a little more time on coarse location). The algorithm is described as follows:

(1) Search for the $\mathrm{x}$-coordinate of the pseudo center: take each point of $\left(\mathrm{x}_{0}\right.$, $\left.\mathrm{y}_{0} \pm 10\right)$ as a temporary center and search for the first white pixel on its left and right side, recording the $\mathrm{x}$-coordinate of the midpoint as the new value $\mathrm{x}$, then take the average of all the new values of $\mathrm{x}$ as the possible $\mathrm{x}$-coordinate of the pseudo center of pupil $\left(\mathrm{x}_{1}\right)$.

(2) Search for the y-coordinate of the pseudo center: take each point of $\left(\mathrm{x}_{1} \pm 10, \mathrm{y}_{0}\right)$ as a temporary center and do as step (1) does, then the coordinate of the pseudo center $\left(\mathrm{x}_{1}, \mathrm{y}_{1}\right)$ could be attained.

(3) Estimate the length of radius of pupil: Take $\left(\mathrm{x}_{1}, \mathrm{y}_{1}\right)$ as the center to calculate the length of chords in some arbitrary directions, and the longest one is thought as the pseudo radius $r_{1}$.

The above method could efficiently reduce the searching range in the following inner boundary fine location, so as to speed it up.

\subsection{Fine Location of the Inner Boundary}

Based on the estimated result, the pupil could be finely located, and the most commonly used formula is (1)

$$
\max _{r, x_{0}, y_{0}}\left|G_{\sigma}(r) \otimes \frac{\partial}{\partial r} \oint_{r, x_{0}, y_{0}} \frac{I(x, y)}{2 \pi r} d s\right|
$$

Formula(1) is a detector of circular edge with $\sigma$ as the scalar, which searches for the optimal solution by iteration of the three-parameter space $\left(\mathrm{r}, \mathrm{x}_{0}, \mathrm{y}_{0}\right)$ to locate the pupil. In this formula, $\left(\mathrm{x}_{0}, \mathrm{y}_{0}\right)$ is the center of the circle; $\mathrm{r}$ is the length of radius of it which ranges from $\left(\mathrm{r}_{1}-10\right)$ to $\left(\mathrm{r}_{1}+10\right) ; \mathrm{G} \sigma(\mathrm{r})$ is a filter usually in the form of Gaussian; and

$\otimes$ is a convolution operation. The essence of the formula is to calculate the average of gray value of every pixel on the circumference of the circles with all the possible 
radii, then to filter the difference between two adjacent circles. Finally, the parameters correspond to the maximum difference is taken as the center and radius of the pupil. The discrete form of the formula is

$$
\max _{n \Delta r_{,}, x_{0}, y_{0}}\left|\frac{1}{\Delta r} \sum_{k}\left[(G((n-k) \Delta r)-G((n-k-1) \Delta r)) \sum_{m} I\left(x_{m, k}, y_{m, k}\right)\right]\right|
$$

In real images, even the gray values of the pixels within the pupil may not be the same, especially in cases that some lighter areas are made because of reflection inside of it under the source of light. The gray values of these areas may be remarkably larger than those of others (Fig. 1(a)). In these conditions, if formula (2) is used, the gray value difference made by these pixels could be more than that of up to 10 regular ones, and so it may lead to the error of locating inside the pupil. This paper detects the circle in binary image, so all the points contribute equally, and this could effectively avoid the problem. The result could be seen in Fig.1(a).

\subsection{Fine Location of the Outer Boundary}

In this paper, the fine location of the outer boundary is based on the inner one. Most of the algorithms utilize circle detectors like formula (2), but in fact, the contrast between the gray values of the outer boundary of iris and sclera (the near to white area outside the iris) are not so remarkable, and the iris has resourceful texture, so it is difficult to locate the outer boundary accurately by those detectors. This paper first uses Canny for edge detection [11,12], and then imposes Hough Voting algorithm on the result to determine its radius and center. Canny algorithm is widely accepted as the best edge detector, which could eliminate the influence of noise more effectively without much loss of true edge information.

In practice, the inner and outer boundary of an iris are not homocentric, and experiments on the CASIA iris database, show that, the vertical difference is within 3 pixels, while the horizontal may be up to 6 pixels. In this paper, all the pixels in the range of $\left[\begin{array}{ll}\mathrm{x} \pm 6 & \mathrm{y} \pm 3\end{array}\right]$ are taken as candidate centers of the outer boundary in Hough voting for circle detection. The detail of Hough voting algorithm is as follows:

(1) Set up an array A, with the dimension of (maximal length of radius of the outer boundary-radius of the inner boundary)*(number of candidates of center of circle, 91 in this paper), and initialize all the elements to 0 ;

(2) Scan the result of Canny edge detection, if a pixel is on the edge, calculate the distance $r$ between it and the candidates of center of circle, all the values of elements correspond to $\mathrm{r}-1, \mathrm{r}, \mathrm{r}+1$ in array A plus 1 ;

(3) Scan array A, and the subscripts correspond to the element with the maximal value is taken as the center and radius of the outer boundary respectively, see Fig. 2.

\section{Exclude Non-iris Areas}

In most cases, the result area after fine location would contain areas of eyelashes or (and) eyelids, if these areas are not removed from the actual ones, the accuracy of iris recognition would be reduced greatly. Many researchers do this by Hough transform, 
modeling eyelids as two parabolic arcs, but this method is very time-consuming and sometimes it is difficult to find the arcs. Basing on the observation that the gray values of the pixels near the outer boundary distribute uniformly, this paper presents a gray value statistical approach on the circumferences of a series of consecutive homocentric circles to obtain two thresholds: $\mathrm{T}_{1}<\mathrm{T}_{2}$, then scans all over the circumference of the outer boundary for further decision. The details of the algorithm are as follows.

(1) Build gray scale histogram of the outer boundary H: From the outer boundary to inside about 4 pixels, select an angle in the left and the right part respectively as the object for statistics, and build its histogram;

(2) Search for the maximum value $\left(\mathrm{N}_{0}\right)$ in the histogram, and record the correspond gray value as $\mathrm{G}_{0}$, then calculate the total number ${ }^{N}=\sum H(i)$;

(3) Search for the upper and lower threshold: Initialize $\alpha$ to a positive real number less than 1.0, then search from $\mathrm{G}_{0}$ to left and right respectively in the histogram, if there are successively 4 gray values correspond to which the number of pixels is less than $\mathrm{N}^{*} \alpha$, stop and record the gray values $\mathrm{T}_{1}<\mathrm{T}_{2}$, calculate the number ${ }^{N_{1}=\sum_{i=T_{1}}^{T_{2}} H(i)}$, if $\mathrm{N}_{1} / \mathrm{N} \geq 85 \%$ or $\mathrm{N} 0 * \alpha<1$, go to (4); Else, decrease $\alpha$, repeat (3);

$\mathrm{T} 1$ and T2 obtained by (3) are taken as the gray level distribution range of the outer boundary of the iris.

(4) Exclude eyelash and eyelid areas: Scan the bottom right circumference from the point of $0^{\circ}$, if there are two consecutive points whose gray values are not in $\left[T_{1}, T_{2}\right]$, stop and record the angle as $-90^{\circ} \leq \theta_{1} \leq 0^{\circ}$; So do the top right, the top left and the bottom left parts, suppose that the angles are $\theta_{2}, \theta_{3}$ and $\theta_{4}$ respectively;

(5) The circular arcs between $\left[\theta_{1}, \theta_{2}\right]$ and $\left[\theta_{3}, \theta_{4}\right]$ are selected as iris areas for following iris recognition, and the remainders are considered to be noniris areas, the result could be seen in Fig.2(c).
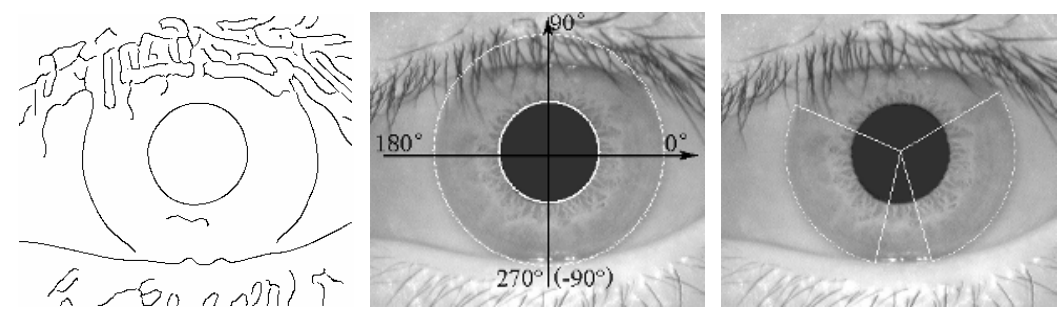

Fig. 2. (a) Result of Canny edge detection (b) fine location result (c)excluding eyelash and eyelid areas

This method could remove certain false areas using the outer boundary information, but how to remove other false inner areas still need careful study, motivated by Canny edge detection, this paper experimented of selecting two thresholds and it works well in some cases but is not so perfect. 


\section{Analysis of Experimental Result}

The iris location algorithm is implemented in $\mathrm{VC}++6.0$. Experiments were conducted using the images in CASIA Iris Image Database 1.0, and they show that the result is satisfactory. In the database, the images are all $320 * 280$, and all the pseudo centers of circle acquired by coarse location are all in the searching range of the next step. Table 1 shows the comparison of the proposed and two commonly used algorithms practiced under the condition of $1.7 \mathrm{GHz}$ CPU 256M RAM. Figure 3 shows some results in experiments.

Table 1. Comparison of accuracy and time of location with other algorithms

\begin{tabular}{lcc}
\hline Algorithms & accuracy & Average time \\
\hline Daugman[1] & $98.4 \%$ & $6.76 \mathrm{~s}$ \\
Wildes[10] & $99.8 \%$ & $8.94 \mathrm{~s}$ \\
proposed & $99.2 \%$ & $0.23 \mathrm{~s}$ \\
\hline
\end{tabular}
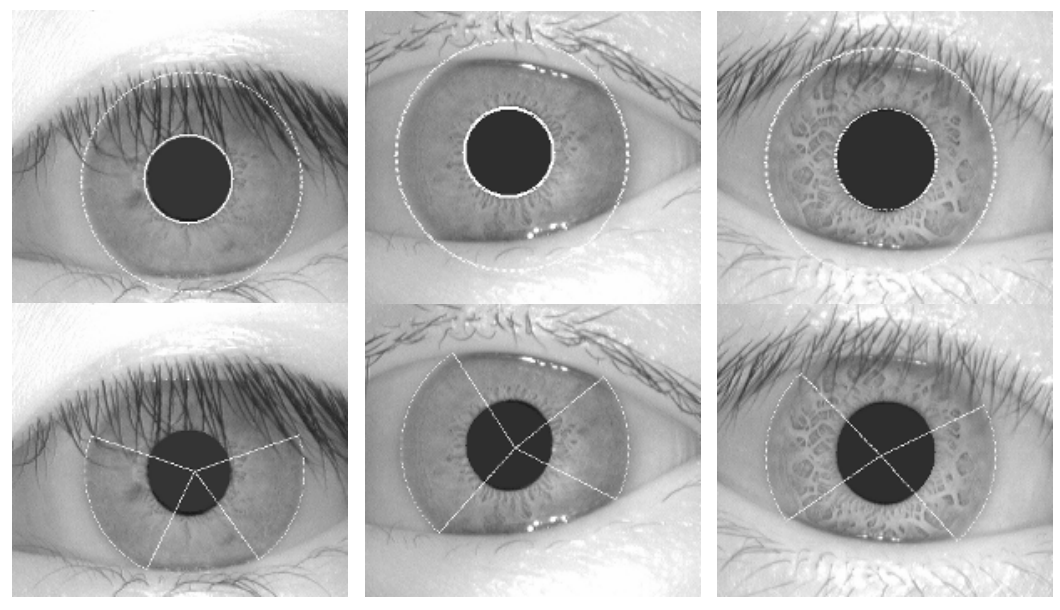

Fig. 3. Some samples of iris location. The above are the results of fine location, and the below ones are correspond results that excluding the eyelash and eyelid areas.

The reduction of time can be contributed to the following factors:

(1) The improved performance of coarse location, so that the searching range could be reduced in the following steps;

(2) Set up an array in advance, storing the offset of $x$ and $y$ correspond to certain length of radii and angles used in fine location, and it could reduce time to $1 / 7-1 / 5$ of previous methods.

In the iris image database (CASIA 1.0), there are about 5 percent of images in which the gray levels of eyelash are lower than those of iris, using the method presented in [8] for coarse location would lead to such result that the located center of 
pupil could be far away from the real one, while uses the method proposed by this paper, the problem could be resolved, and the fine location time could also be decreased. Compared to the commonly used J.Daugman's iris location algorithm, it could effectively avoid the error location problem caused by reflection inside the pupil. And combines Canny edge detection and Hough voting mechanism for location of the outer boundary could ensure satisfactory performance under the condition of very clear texture.

\section{Acknowledgements}

This work was supported by the Natural Science Foundation of China (Grant No. (60433020)) and the Key Laboratory for Symbol Computation and Knowledge Engineering of the National Education Ministry of China. (93K-17).

All the images appeared in this paper were supplied by the Institute of Automation, Chinese Academy of Sciences, the authors would like to thank them.

\section{References}

[1] J.Daugman: High confidence visual recognition of persons by a test of statistical independence. IEEE Transactions On Pattern Analysis and Machine Intelligence, 15 (1993) 1148-1161

[2] Wang Chengru, Hu Zhengping: Iris Location Algorithm Based on Geometric Features. Journal of Image and Graphics A, 8 (2003) 683-685

[3] Fan Kefeng, Zeng Qingning: A Research on Iris Location Algorithm, Computer Engineering and Applications, 40 (2004) 60-61

[4] Yang Wen, Yu Li etc: A Fast Iris Location Algorithm. Computer Engineering and Applications, 40 (2004) 82-84

[5] Richard P. Wildes: Iris Recognition: An Emerging Biometric Technology. Proceedings of the IEEE, 85 (1997) 1348-1363

[6] Jiali Cui, Li Ma, etc: An Appearance-based Method for Iris Detection, ACCV2004, 2: 1091-1096

[7] Yuan Weiqi, Ma Junfang etc: A New Method of Iris location based on Active Contour. Computer Engineering and Applications, 39 (2003) 104-107

[8] Xue Bai, Liu Wenyao etc: Research on Iris Image Preprocessing Algorithm. Journal of Optoelectronics • Laser, 14 (2003) 741-744

[9] Xiaoyan Yuan And Pengfei Shi: An Iris Segmentation Procedure for Iris Recognition. Advances in Biometric Person Authentication, 5th Chinese Conference on Biometric Recognition, SINOBIOMETRICS 2004.12:546-553

[10] Chen Gong, Zhou Youling: Iris Location Based on Hough Transform. Journal of East China University of Science and Technology, 30 (2004) 230-233

[11] Wan Li, Yi Ang, Fu Ming: An improved edge-detection method based on Canny algorithm. Computing Technology and Automation, 22 (2003) 24-26

[12] Zhang Xiaohong, Yang Dan, Liu Yawei: Improved edge detection algorithm based on Canny operator. Computer Engineering and Applications, 39 (2003) 113-115

[13] CASIA Iris Image Database, http://www.sinobiometrics.com 